\title{
A Mass Spectrometry Imaging Study of Lipid Differentiation in Model Systems
}

\author{
Emily Schenk ${ }^{1}$, Michael Smotherman ${ }^{2}$, Caurnel Morgan ${ }^{3}$, and Francisco Fernandez-Lima ${ }^{1}$ \\ 1. Department of Chemistry and Biochemistry, Florida International University \\ 2. Department of Biology, Texas A\&M University \\ ${ }^{3}$ Department of Nutrition and Food Science, Texas A\&M University
}

Mass spectrometry imaging has become the method of choice for chemical mapping of organic and inorganic compounds from various surfaces, in particular for tissue sections.[1] Three techniques are emerging as good candidates: MALDI-MS, nano-SIMS, and TOF-SIMS. In MALDI-MS, molecular ion identification and localization with a $50 \mu \mathrm{m}$ spatial and nanomolar sensitivity can be achieved over a wide mass range (typically, $400-40 \mathrm{kDa}$ ), while analysis reproducibility depends strongly on the sample preparation method and matrix deposition. That is, the matrix remains a crucial choice for the chemical class to be studied in MALDI experiments and a number of new matrixes have been developed.

TOF-SIMS can detect molecular ions with a spatial resolution of $500 \mathrm{~nm}$ to a few micrometers. The selection of the primary ion in a TOF-SIMS experiment defines the mass range of the analysis. For example, monatomic projectiles (e.g., In, Ga, and $\mathrm{Cs}$ ion sources) induce high surface damage and low intact molecular ion emission, leading mainly to the detection of fragment ions (e.g., head groups in the case of lipid samples).[2] When small cluster ion sources (e.g., $\mathrm{Au}_{3}{ }^{+}$and $\mathrm{Bi}_{3}{ }^{+}$) are used TOF-SIMS can also generate molecular signals in the 1000 - 1500 mass range. Moreover, the use of larger cluster projectiles (e.g., $\mathrm{C}_{60}$ and $\mathrm{Au}_{400}$ ) with TOF-SIMS for surface analysis and characterization has shown significant advantages due to the enhanced emission of molecular ions and reduced molecular fragmentation.[3] With temporally and spatially discrete cluster impacts, the small interaction volume $\left(\sim 10^{3} \mathrm{~nm}^{3}\right)$ and large ionized ejecta makes these massive probes promising candidates for surface molecule interrogation.

Current challenges are in the development of new TOF-SIMS probes capable of generating signature secondary ions from a surface of interest. Recent efforts have focused on the use of higher energy and larger cluster projectiles (e.g., $\mathrm{C}_{60}$ and $\mathrm{Au}_{400}$ ) to increase the secondary ion signal. For example, three main features can be observed from the comparison of the secondary ion emission profiles from polyatomic and nanoparticle projectiles in the case of lipid analysis from tissue sections: (i) similar analyte-specific fragment ions, (ii) enhanced molecular ion emission for the case of nanoparticle projectiles impacts compared with polyatomic impacts (near two orders), and (iii) abundant molecular ion emission of the lipid components for nanoparticles projectiles $\left(10^{-1}-10^{-2}\right.$ molecular ions per impact).

In the present work, we will show the potentialities of mass spectrometry based imaging for the analysis of lipid distributions form model systems as a function of a biological question (e.g., disease vs. non disease, low vs. high fatty acid diet, etc.). The analysis was performed using a combination of MALDI, polyatomic and clusters ion sources coupled to mass spectrometry. In the case of brain tissue sections, we attribute the high lipid abundance as compared with other chemical classes to the fact that lipids account for up to $50 \%$ of the dried weight of brain tissue sections. Comparison between positive and negative MS mode shows that molecular ion emission can be directly correlated to the surface concentration and the ionization probability. In particular, two distinct lipid distributions are observed for positive and negative MS modes, which can be attributed to the difference in the charge carriers 
(lipid head groups) for the different lipid classes.

References:

[1] P. Chaurand, S. A. Schwartz, M. L. Reyzer, and R. M. Caprioli, Toxicologic Pathology 33 (2005) 92.

[2] M. L. Pacholski, D. M. Cannon, A. G. Ewing, and N. Winograd, Rapid Communications in Mass Spectrometry 12 (1998) 1232.

[3] F. A. Fernandez-Lima, J. Post, J. D. DeBord, M. J. Eller, S. V. Verkhoturov, S. Della-Negra, A. S. Woods, and E. A. Schweikert, Anal. Chem. 83 (2011) 8448.

Bat Brain

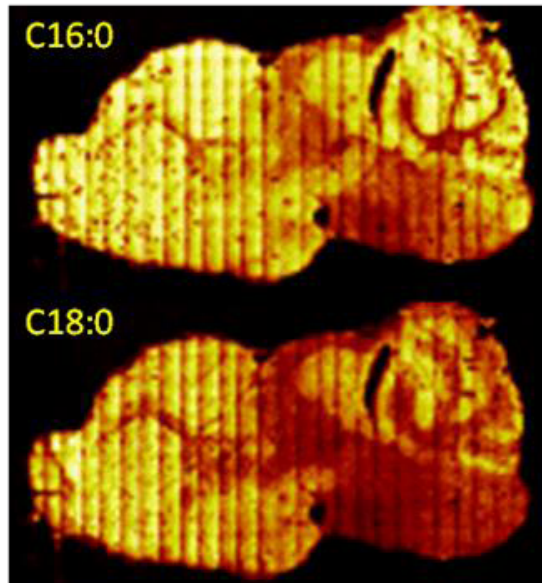

ST24:1

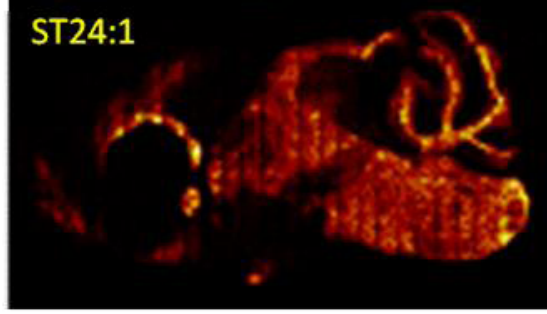

\section{Mouse Brain}

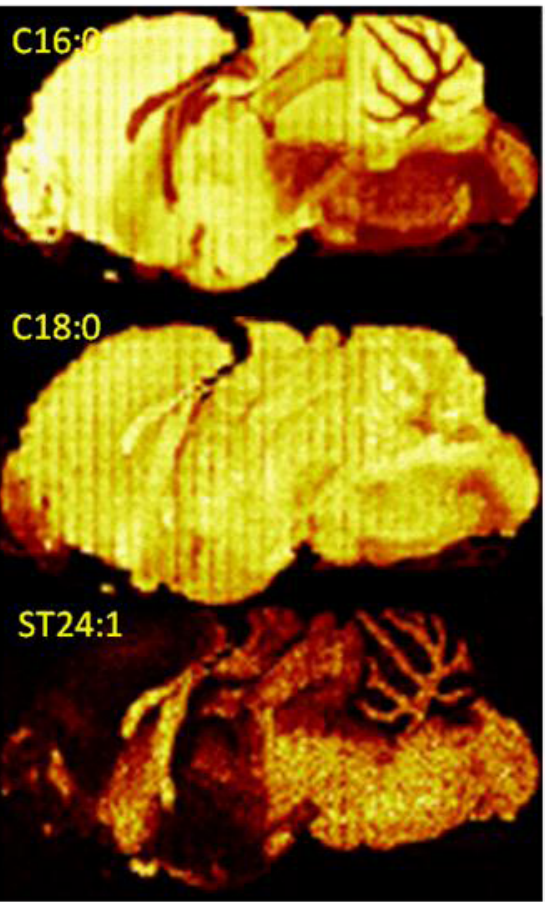

Figure 1. Negative mode MS molecular ion distribution of C16:0 and C18:0 fatty acid and ST24:1 lipid components for the case of a bat and mouse brain tissue section obtained with ToF-SIMS $\left(\mathrm{Bi}_{3}{ }^{+}\right)$ 